\title{
Robust Locator Layout Design for B-pillar Based on Taguchi Experiment XIA Yu-feng ${ }^{1, a}$, Ren $\mathrm{Li}^{1, \mathrm{~b}}$, YE Cai-hong ${ }^{1, \mathrm{c}}$ Wang $\mathrm{Li}^{1, \mathrm{~d}}$
}

\author{
School of Material Science and Engineering, Chongqing University, Chongqing 400044, China \\ arenli1991@cqu.edu.cn, b20130902005@cqu.edu.cn, cwilly@ cqu.edu.cn, dxuqiang223@ 163.com
}

Keywords: assembly deviation; locator layout; Taguchi orthogonal experiment; B-pillar; 3DCS

\begin{abstract}
For solving the existing problems of assembly quality of B-pillar, a robust design approach was utilized to optimize locator layout of Reinf Pillar and Side Beam Threshold so that the assembly deviation of B-Pillar was minimized. Taguchi orthogonal experiment was carried out to perform the idea by taking the locators coordinate as design parameters, taking the manufacturing and positioning deviation of parts as noise factors. The gap value between Reinf Pillar and Side-body Panel was considered as the measurement index. Furthermore, based on the establishment of robust evaluation function and signal-to-noise ratio formula, the robust layout scheme was determined. The 3DCS(Dimensional Control Systems), a tolerance simulation software, was running, and the simulation analysis results show that robust design greatly reduces the assembly gap, effectively solving the problem of large assembly deviation and quality fluctuation of B-Pillar.
\end{abstract}

\section{INTRODUCTION}

Accompanied with the rapidly development of automobile industry, there are tremendous needs to further expand the technique to make the assembly quality more precise, particularly the quality of B-Pillar, which has an enormous impact on the car's wind noise, sealing and security issues[1]. Now-days the pivotal segment of B-pillar assembly is the Reinf Pillar and Side Beam Threshold for the reasons of their complicated shape and sophisticated surface profile. Traditional assembly mostly rely on experience, which inevitably led to greater error accumulation [2]. Along with the presence of an increasing number of assembly quality problems, robust design has attracted more attention as an effective method of quality engineering [3]. The purpose of robust design is to find a set of parameters that are not sensitive to noise factors, so that the product quality is stable and small fluctuations [4].

In recent years, there were lots of researches about the quality robustness design. Huang et al. [5] studied the robustness of multi-station assembly by using the continuous space filling method. Cai et al. [6] put forward an optimization method of pins layout for the positioning process of sheet metal, which can remarkably decreased assembly deviation. Cao et al. [7] proposed robust tolerance design as tolerance design always not take the impact of noise factors into consideration. Most of the above researches were aimed at robust design of previous tolerance allocation. However, changing tolerance would inevitably involve adjustment of the mold and technology update, resulting in cost increasing.

In view of the problem, this paper presented the method of robust locator layout design, which took the locators on Reinf Pillar and Side Beam Threshold as object. Then determined the levels of locators' coordinate values by establishing the influence curve between coordinates and the deviation of measurement point [8]. Combined orthogonal experiment with 3DCS software, the best levels of these locators were found out. Finally, the assembly quality of B-Pillar assembly is better, providing a certain guidance for the follow-up actual production.

\section{II.THE INTRODUCTION OF LOCATING PRINCIPLE}

The common 3-2-1 locating principle was usually adopted during the assembly process of rigid panel parts $[9,10]$. In general, the principle can be described from two aspects, which includes two locating pins and three locating blocks. The two locating pins constraint plane in the three degrees 
of freedom while three locating blocks limit the other freedoms of part [11]. Among the two locating pins, the 4-way pin controls movements along $\mathrm{X}$ and $\mathrm{Z}$ directions while the 2-way pin only restricts rotation by Z-direction. In spite of the widely used of 3-2-1 principle, the $\mathrm{N}-2-1(\mathrm{~N} \geq 3)$ principle where " $\mathrm{N}$ " represents the number of the locating blocks came into use in compliant parts with large size and insufficient stiffness for the purpose of ensuring the equilibrium [12]. A typical example of N-2-1 locating principle, which has four locating blocks, namely 4-2-1 principle. Locating blocks and pins, normally labeled as locators, are the most common fixtures for the assembly of sheet panels [13].

It goes without saying that any variation in the two elements will translate into a faulty positioning of work-piece thus resulting in assembly error. However, it is shown that the deviation of locating blocks and pins have quite different influence: the deviation of the pins cause rigid body motion whereas locating blocks cause deformation of parts [14].

The 3D structural model of B-Pillar was shown in Figure 1. Reinf Pillar and Side Beam Threshold are flexible sheet metal parts, so the 4-2-1 locating principle was adopted in assembly. The auto-bend commands in 3DCS can simulate 4-2-1 assembly. L1, L2, L3, L4, L5, L6, L7 and L8 are respectively on behalf of the positions of the locating blocks on the two parts. P1 and P2 are the position of the two pins. $\mathrm{P}_{\mathrm{r}}$ is the measurement point. This paper was mainly aimed at robust layout design of these locating blocks.

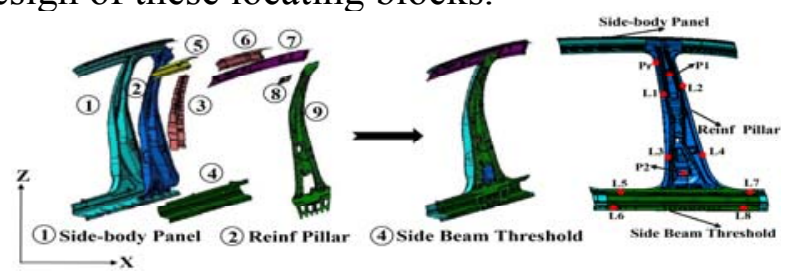

Fig. 1 Three-dimensional assembly model of B-pillar

\section{III.Taguchi orthogonal experiment design}

A. Coordinate Values Analysis

In order to get a reasonable range of coordinate values of blocks L1-L8, the coordinates were regarded as independent variables, and the deviation of the point Pr along different directions were regarded as the dependent variable. The 3DCS software was used to simulate assembly, and the ORIGIN software to fit the results of simulation. Finally the relationship between the two variables were obtained in the forms of graph [15]. Limited to the space, only the graphs of L3 and L4 were displayed in Fig. 2.
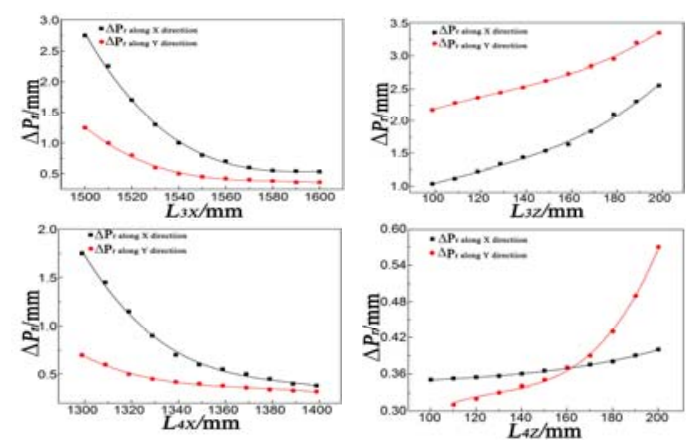

Fig. 2 Relationship between $\Delta \operatorname{Pr}$ and coordinates

"Lab" in the graph indicates the "b" direction of the block "a". $\Delta \operatorname{Pr}$ means the deviation of Pr. From the graph above, it is not difficult to find that the coordinates changes along different directions have diverse influence on the deviation of point Pr. For example, the changes in $\mathrm{Z}$ direction of L1 and L2 had great influence while there was no obvious effect on X direction. Nevertheless, both changes in X \& Z direction of L3 and L4 made big difference.

In the experiment design, more attention should be taken to the locating blocks which had significantly effect on the deviation. The coordinate levels should be also close to the region that was sensitive to assembly deviation. On the contrary, the region did not affect obviously should take 
no value or less value.

B. Orthogonal Experiment Design

Through the analysis on the graph, the orthogonal experiment table was built up by taking three values in $\mathrm{Z}$ direction for L1 and L2, two values in X and Z direction for L3 and L4 respectively as displayed in Table 1.

TABLE 1 Levels design of locator layout parameters

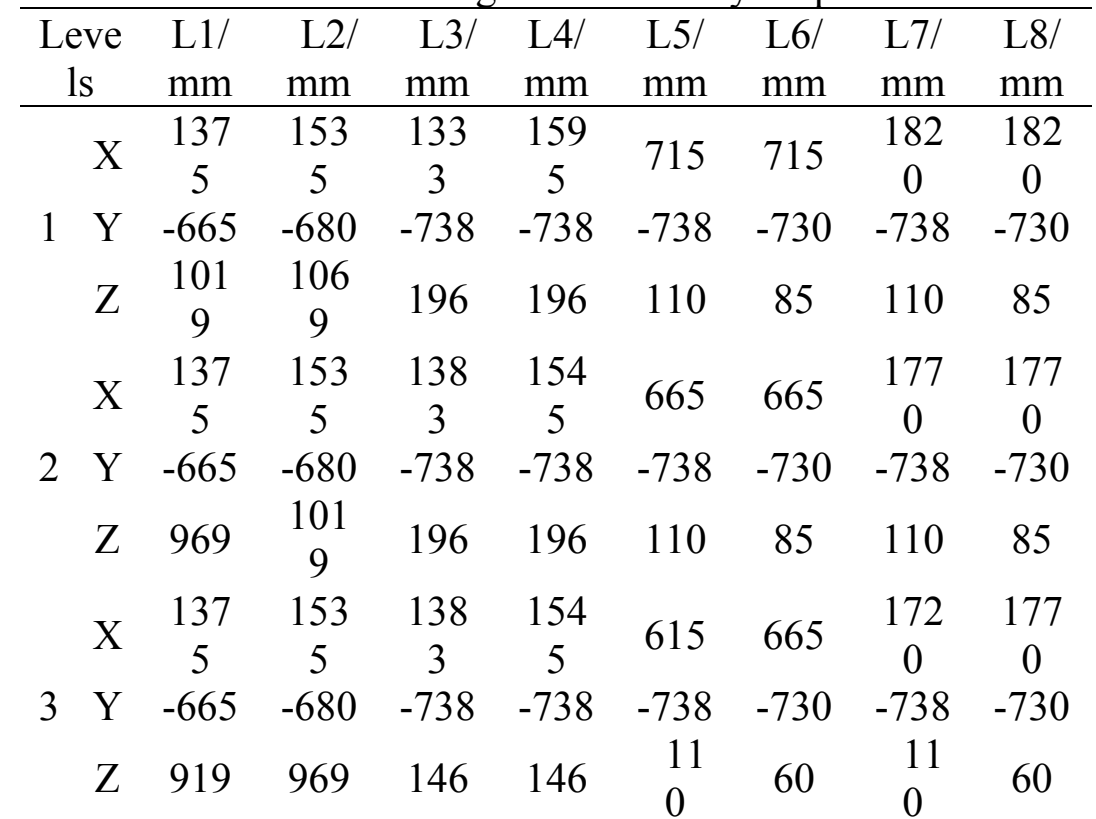

The main factors affecting assembly quality were the locating deviation and the manufacturing deviation of locating blocks, which were considered as noise factors. This study was linked with 14 noise factors, respectively involving the manufacturing deviation of the eight locating blocks and the locating deviation of L1, L2, L3, L4, L6 and L8. In order to build orthogonal experiment table of the noise factors, each factor was given two levels. The level values were completely synchronized with each other an $d$ the deviation sources obeyed normal distribution. In this paper, as the layout parameters were eight factors three levels and the noise parameters were fourteen factors two levels, it is appropriate to choose $\mathrm{L}_{16}\left(2^{15}\right)$ as the outer orthogonal table as shown in Table 2 and choose $\mathrm{L}_{27}\left(3^{13}\right)$ as the internal table as shown in Table 3.

TABLE 2 Levels of noise factors

\begin{tabular}{ccc}
\hline \multirow{2}{*}{$\begin{array}{c}\text { eev } \\
\text { el }\end{array}$} & \multicolumn{2}{c}{ Noise factors } \\
\cline { 2 - 3 } & $\begin{array}{c}\text { Manufacturing } \\
\text { deviation }\end{array}$ & $\begin{array}{c}\text { Locating } \\
\text { Error }\end{array}$ \\
\hline 1 & $\mathrm{~N}(0,0.36)$ & $\mathrm{N}(0,0.36)$ \\
2 & $\mathrm{~N}(0,0.64)$ & $\mathrm{N}(0,0.64)$ \\
\hline
\end{tabular}


TABLE 3 Orthogonal experiment design of locator layout

\begin{tabular}{|c|c|c|c|c|c|c|c|c|c|}
\hline \multirow{2}{*}{$\begin{array}{l}\text { Expe } \\
\text { rime } \\
\mathrm{nt}\end{array}$} & \multicolumn{8}{|c|}{ Factors } & \multirow{2}{*}{$\mathrm{R}^{\mathrm{SN}}$} \\
\hline & & I2 & $\mathrm{I3}$ & $\mathrm{I} 4$ & I5 & I6 & 17 & 18 & \\
\hline 1 & 1 & 1 & 1 & 1 & 1 & 1 & 1 & 1 & $\begin{array}{l}10.907 \\
5\end{array}$ \\
\hline 2 & 1 & 1 & 1 & 1 & 2 & 2 & 2 & 2 & $\begin{array}{l}10.833 \\
7\end{array}$ \\
\hline 3 & 1 & 1 & 1 & 1 & 3 & 3 & 3 & 3 & $\begin{array}{l}10.923 \\
7\end{array}$ \\
\hline & $\cdot$ & $\cdot$ & $\cdot$ & $\cdot$ & & 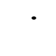 & $\cdot$ & & . \\
\hline 25 & 2 & 1 & 2 & 3 & 3 & 1 & 2 & 3 & $\begin{array}{l}10.907 \\
2\end{array}$ \\
\hline 26 & 2 & 2 & 3 & 1 & 1 & 2 & 3 & 2 & $\begin{array}{l}10.936 \\
1\end{array}$ \\
\hline 27 & 2 & 2 & 3 & 1 & 2 & 3 & 1 & 3 & $\begin{array}{c}10.911 \\
3\end{array}$ \\
\hline
\end{tabular}

\section{Results and discussion}

\section{A. Signal-to-Noise Ratio}

Robustness, known as one of the indicators of assembly quality, refers to the insensitivity of small impact on the dependent variable when deteriorated factor condition occurs. Robust design was firstly put forward by Japanese Taguchi, which focuses on the quality of design phase rather than the manufacturing. The signal-to-noise ratio, as is so-called SNR, was proposed as a method of measuring the quality characteristics of product in robust design. Since it is the ratio of signal and the amount of noise, it implies that larger SNR leads to better robustness [2]. For this assembly, the gap value between Reinf Pillar and Side-body panel was regarded as the assembly quality metrics of B-pillar. Three groups of the measuring points between them called $d 0, d 1, d 2$ whose nominal value is $0 \mathrm{~mm}$ were chosen as the measurement shown in Figure 3. There were total 432 deviation models built using the analyst module of 3DCS as the sum of the 27 kinds of locators layout corresponding to 16 kinds of the bias source programs [8]. Following the simulation, the mean and variance value of the statistical characteristics about $d 0, d 1$ and $d 2$ under each layout scheme were calculated. Accordingly, the robustness evaluation function of assembly quality was established as below:

$$
Y=\bar{\mu}^{2}+\bar{\sigma}^{2}
$$

In robust design, $Y$ is the smaller-the-better function. Its value is hoped to be as small as possible. Supposing $Y \sim N\left(\mu, \sigma^{2}\right)$, it is equivalent to that $\mu$ and $\sigma^{2}$ are expected to be smaller. So the SNR calculation formula of quality robustness was presented in the following:

$$
S N R=10 \lg \frac{n}{\sum_{i=1}^{n} y_{i}{ }^{2}}
$$

Where, $\mathrm{n}$ denotes the number of experiment schemes of noise factors, taking 16 in this paper. Afterwards, the SNR value of each experiment was finally figured out by substituting the obtained values of $y_{i}$ in (2). 


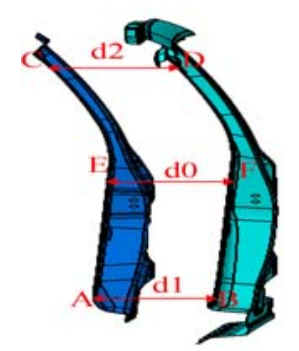

Fig. 3 Measurement between side-body panel and Reinf Pillar

\section{B. Simulation Results Analysis}

Considering all the details during the initiation of assembly would result in the 3D-model requiring prohibitive computing time to handle for simulation. Therefore, appropriate assumption have been made to simply the problem by taking the statistical analysis of 2000 assemblies as one simulation result in 3DCS. The assembly eligibility criteria was limited within the range $6 \sigma$ to tolerances, which referred to the percent of eligibility reached to $99.73 \%$ and the super rate (Tot-OUT \% ) of the measurement was less than 5\% [15].

Under this hypothesis, the simulation was carried on to acquire the measurement value. By the calculation of numerical simulation results under different schemes, the SNR values were presented in Table 3. In order to estimate the influence of different factors, range analysis was executed and the result was summarized in Table 4.

TABLE 4 Range analysis of assembly quality

\begin{tabular}{ccccccccc}
\hline $\begin{array}{l}\text { Fac } \\
\text { tors }\end{array}$ & L1 & L2 & L3 & L4 & L5 & L6 & L7 & L8 \\
\hline \multirow{2}{*}{ K1 } & 98.0 & 98.0 & 98.2 & 98.1 & 98.1 & 98.2 & 98.1 & 98.0 \\
& 457 & 864 & 050 & 966 & 672 & 059 & 983 & 728 \\
K2 & 98.2 & 98.1 & 98.1 & 98.1 & 98.1 & 98.0 & 98.1 & 98.1 \\
& 544 & 846 & 290 & 996 & 590 & 805 & 483 & 138 \\
K3 & 98.2 & 98.2 & 98.1 & 98.1 & 98.1 & 98.2 & 98.1 & 98.1 \\
& 080 & 371 & 724 & 119 & 819 & 217 & 615 & 215 \\
& 0.20 & 0.15 & 0.07 & 0.08 & 0.02 & 0.01 & 0.05 & 0.04 \\
$R$ & 87 & 07 & 60 & 96 & 29 & 58 & 00 & 87 \\
\hline
\end{tabular}

The analysis shows that:

(1)K1, K2, K3 respectively expressed the sum of SNR under three levels of the factors, and R, for the range. The $\mathrm{K}$ value indicates assembly robustness, that's to say, the larger the $K$ value, the more robust the assembly. Analyzing the table data, we can easily draw the conclusion that the best assembly was under the level 2 of L1, level 3 of L2, level 1 of L3, level 2 of L4, level 3 of L5, level 3 of L6, level 1 of L7, level 3 of L8. It was because that under these level combinations, the locator layout would be more uniform and insensitive to noise factors. Any level changes would make the assembly worse. So this was the robust deign scheme for Reinf Pillar and Side Beam Threshold.

(2)Bigger $\mathrm{R}$ value means a large diversity among three levels of locator, also poor resistance to sensitivity. Compared the R-value of Reinf Pillar with that of Side Beam Threshold, it was obviously to discover that $R 1, R 2, R 3$ and $R 4$ were a value bigger than $R 5, R 6, R 7$ and $R 8$. So the locators on Reinf Pillar tended to have a greater influence than Side Beam Threshold. It was because that the assembly of Reinf Pillar was in the middle phrase, connected with four parts, so the effect of accumulate error would occupy a large part. In the actual production, more attention should be focused on the deviation and tolerance control of Reinf Pill ar.

(3)In view of all these $R$ values, it can be concluded that the locating blocks which were close to the measurement point have stronger effect on the assembly than others. For instance, the R-value of L1 and L2 respectively reached to 0.2087 and 0.1507 , greatly exceeded the others. Consequently, the locators tolerance closed to measuring point should be key monitored during the production process. 


\section{Comparison of Different Schemes}

The original layout scheme was experience value without any calculation, which was consist of the level 1 of each factor. It was widely used as the lack of method to choose more suitable locators. In this case, robust design provided approach to optimize locator layout. A comparison was made between original scheme and robust scheme under sixteen groups of noise factors. The average values related to $\mathrm{d} 0, \mathrm{~d} 1, \mathrm{~d} 2$ were compared and analyzed, as shown in Table 5 .

TABLE 5 Numerical results comparison of initial and robust design

\begin{tabular}{lccccc}
\hline $\begin{array}{l}\text { Schem } \\
\mathrm{e}\end{array}$ & Result & $d 0$ & $d 1$ & $d 2$ & Average \\
\hline & $\begin{array}{c}\text { Nominal } \\
\text { mm) }\end{array}$ & 0 & 0 & 0 & 0 \\
$\begin{array}{l}\text { Origin } \\
\text { al } \\
\text { design }\end{array}$ & Mean(mm) & 0.2315 & 0.2601 & 0.3715 & 0.28777 \\
& 6 & 0.3126 & 0.3316 & 0.3518 & 0.28470 \\
& Tot-OUT\% & 8.05 & 8.15 & 8.45 & 8.22 \\
& Nominal( & 0 & 0 & 0 & 0 \\
Robust & Mean(mm) & 0.1345 & 0.1596 & 0.2451 & 0.17981 \\
design & 7 & 9 & 6 & \\
& 6 & 0.1716 & 0.1712 & 0.1530 & 0.21310 \\
& Tot-OUT\% & 8 & 5 & 7 & 3.18 \\
\hline
\end{tabular}

According to the calculation results in Table 5, the quality fluctuation of original design was $10.9 \%$, but only $4.9 \%$ of robust design. The gap $\mathrm{d} 0, \mathrm{~d} 1$ and $\mathrm{d} 2$ reduced by $41.9 \%, 38.9 \%$ and $34.1 \%$. The super rate (Tot-OUT \%) decreased by 59\%, 61.3\% and $63.3 \%$.

\section{Conclusions}

This paper was focused on the impact on final assembly caused by different layouts of locating blocks. Taguchi orthogonal experiment was used to arrange the whole scheme design. 3DCS was the main software to conduct tolerance simulation. Based on the help of software, the robust locator layout scheme was finally obtained through experiment design, analysis, and result optimization. Compared with the original design scheme, robust design has obvious advantages in improving assembly quality and decreasing quality fluctuation. At the same time, the combination of Taguchi experiment with 3DCS software provides a new approach for the low cost and high quality production of B-Pillar. However, the parts of B-Pillar are complex, and there are interactions between different levels of assembly, so the robust research on the locator layout of the whole B-Pillar is more practical.

\section{ACKNOWLEDGMENT}

The author acknowledges that support was received from the Fundamental Research Funds for the Central Universities (No. CDJZR13130082).

\section{REFERENCES}

[1]S. Du, L. Xi, J. Ni, Product lifecycle-oriented quality and productivity improvement based on stream of variation methodology [J]. Computers in Industry, 2007, 6(23): 1-13.

[2] Q. Huang, J. Shi. Part dimensional error and its propagation modeling in multi-operational machining process [J]. Journal of Manufacturing Science and Engineering, 2007, 125(2): 255-262. 
[3] C. Stephane, F. Bennis, P. Wenger. Tolerance synthesis of mechanisms: A robust design approach [J]. Journal of Mechanical Design, 2005, 127(15): 86-94.

[4] Y. Ding, J. Jin, D. Ceglarek, et al. Process-oriented tolerance for multi-station assembly systems [J]. IIE Transactions, 2005, 37(5): 493-508.

[5] W. Z. Huang, J. Lin, M. Bezdecny, et al. Stream of variation modeling-Part I: A generic three-dimensional variation model for rigid-body assembly in single station assembly process [J]. Journal of Manufacturing Science and Engineering, 2007, 129(4): 821-831.

[6] W. Cai. Robust pin layout design for sheet-panel locating [J]. The International Journal of Advanced Manu-fracturing Technology, 2006, 28(5): 486-494.

[7] Y. L. Cao, Y F Wang, J X Yang, et al. Study on feasibility robust tolerance design method [J]. Journal of Zhejiang University: Engineering Science, 2005, 39(293): 364-367.

[8] Z. J. Wen, F Zhang, H C Liu et al. Robust pin hole layout design for car light cover assembly based on Taguchi orthogonal experiment and 3DCS [J]. Chinese Journal of Automotive Engineering, 2013, 3(4): 275-281.

[9] P. Kim, Y. Ding. Optimal Design of fixture layout in multi-station assembly processes [J]. IEEE Transaction Automation science and Engineering, 2004, 1(2): 133-145.

[10] W. Huang, Z. Y. Kong, A. Chennamaraju. Robust Design for fixture layout in multi-station assembly systems using sequential space Filling Methods [J]. Journal of Computing and Information Science in Engineering, 2010, 10(4): 1-11.

[11] A. Jaime, S. Camelio, S. Jack Hu. Impact of fixture design on sheet metal assembly variation [J]. Journal of Manufacturing System, 2010, 23(3): 182-193.

[12] N. Kaya. Machining fixture locating and clamping position optimization using genetic algorithms [J]. Computers in Industry, 2006, 57(2): 112-120.

[13] S. Vishnupriyan, M. C. Majumder, K. P. Ramachandran. Optimization of machining fixture layout for tolerance requirements under the influence of locating errors [J]. International Journal of Engineering, Science and Technology, 2010, 2(1): 152-162.

[14] S.Zhou, Q. Huang, and J. Shi. State Space Modeling of Dimensional Variation Propagation in Multistage Machining Process Using Differential Motion Vectors [J]. IEEE Transactions on Robotics and Automation, 2003, 19(2): 296-309.

[15]L. Zhang, X. H. Wei, Y. Yin et al. Tolerance analysis for the landing gear of one large-lap aircraft based on 3DCS [J]. Journal of Machinery Design\& Manufacture, 2012, 12(7): 73-75 (in Chinese). 